\title{
Innovative wheel tread design aimed to tramcar- track interaction improvement when passing curves of a small radius
}

\author{
Vladimír Hauser ${ }^{1, *}$, Olena S. Nozhenko ${ }^{1}$, Kateryna O. Kravchenko ${ }^{1}$, Mária Loulová ${ }^{1}$, \\ Juraj Gerlici ${ }^{1}$, Tomáś Lack ${ }^{1}$ \\ ${ }^{1}$ University of Žilina, Faculty of Mechanical Engineering, Department of Transport and Handling \\ Machines, Univerzitná 1, 01026 Žilina, Slovak Republic
}

\begin{abstract}
Tramways in cities often ride in track curves of a small radius, which is followed by an increased effect of the vehicle on the track. In the rail - wheel contact often occurs creep in longitudinal as well as in lateral direction, which causes increase of riding resistance, wear in rail - wheel contact and noise operation. Exactly with the aim to reduce these undesirable effects we designed a tram bogie with steered wheelsets and with the double wheel tread. This inventions are registered by authors under Utility Model Nr. u201609015 and Patent Application Nr. a201701589. Description of proposed process of passing vehicle through strongly curved track is given in this paper. A comparison of creep velocities in rail - wheel contact results obtained by computer multibody simulations for a tram vehicle riding through a Scurve are presented. Purposefulness of proposed invention is investigated by constructing vehicle ride resistance dependence to the track curve radius.
\end{abstract}

Keywords: double wheel tread, strongly curved track, tramcar throughputness

\section{Introduction}

Special attention is taking place in the environment of public transport railways, for which higher amount of radius curves up to 17 meters being applied is specific. The outcome of such operation of vehicles is an increase in vehicle's effects on the track in the rail-wheel contact resulting in increased ride resistance, creep in the rail-wheel contact patch, speeding up the process of wear in the contact-pair as well as noise generation. At present, a variety of technical solutions for the vehicle bogie design as well as rail designs focused on decreasing of these negative effects exists [1-5]. Their use in smaller radius curves however, cannot give acceptable results and often causes complications in bogie design. The authors give a concept of creep reduction in rail-wheel contact by double wheel tread, which doesn't require complicated bogie design.

Creep in wheel-rail contact takes place in both lateral and longitudinal direction. Creep in lateral direction in small radius curves reaches higher values. It is possible to effectively minimize these values by using a bogie design, which allows steering of the wheelsets to a

*Corresponding author: vladimir.hauser@fstroj.uniza.sk

Reviewers: Robert Grega, Milan Nad' 
radial position $[6,7]$. The authors aim at a design solution, which would remove the cause of longitudinal creep in rail-wheel contact and that would not be complicated at the same time. We suggest solving this task by using a double wheel profile and a change in rail geometry of a small radius curve [8]. The purposefulness of this solution is proven by simulation analysis of the vehicle ride dynamics.

\section{Motivation}

Vehicle forces affect the track through contact between wheels and rails. The effect is especially intensive when the vehicle passes through arcs of small radius. Often it is not possible to transfer it through the wheel-rail adhesion bond, and so a creep that takes place in the longitudinal and lateral direction develops. [9] Since the creep is present in the contacts and the shear friction coefficient is not zero, part of the vehicle performance serves to overcome the mentioned creep. The dependence of this power dissipation from the radius of the track curve gives an overall view on the characteristics of the vehicle ride through a track curve.

This dependence, as shown in Fig. 1, was obtained for a $10 \mathrm{~km} . \mathrm{h}-1$ speed of a vehicle similar to a T3 tram [10], with values for two types of bogies and two types of wheel profiles being presented. The first bogie has fixed wheelsets, the second bogie is equipped with a mechanism for steering the wheelsets. The E-99-00 wheel profile has lower tread conicity values, the KP-1 profile [11] has its conicity adjusted to tracks with a greater number of curves. In all cases, the power dissipation of the vehicle is divided into a component covering creep in the longitudinal and lateral directions.
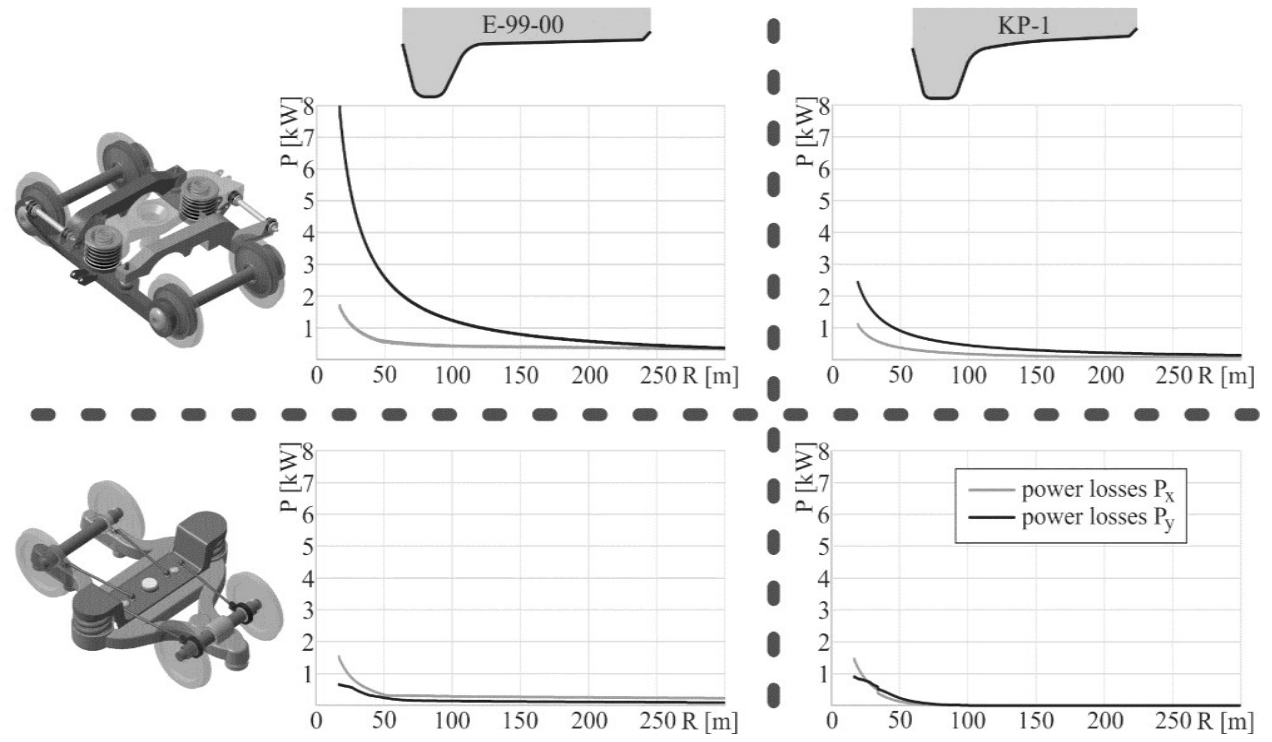

Fig. 1. Comparison of the power dissipation of two bogie types equipped with two different wheel profiles

From the comparison of the power dissipation of the vehicle shown in Fig. 1 it can be understood, that the wheelset steering mechanism can effectively minimize creep in the lateral direction particularly, even when a wheel profile not suitable for ride through track curves is used. However, even in the case of a vehicle equipped with wheelset steering mechanism with a wheel profile suitable for ride through track curves, the power dissipation in small radius curves reaches values about $2 \mathrm{~kW}$. 
The ultimate product of this power dissipation is the wear of the wheel-rail contact. The wear range is cumulative in relation to the number of passes of the vehicle at the given track section. It is therefore particularly desirable to minimize the power dissipation of the vehicle, thereby prolonging the operating life of the contact wheel-rail pair. We suggest to reach this state by minimizing the longitudinal creep.

\section{Analysis of the wheelset ability to pass through a track curve}

Curve radius, at which the wheelset is theoretically able to ride through without creep can be determined using the equation:

$$
\mathrm{R} \geq \frac{2 \mathrm{Sr}}{\Delta \mathrm{r}}[\mathrm{mm}]
$$

where $2 S$ is contact circles distance [mm], $r$ is wheel radius [mm], $\Delta r$ is the value of the wheel profile delta function at the moment the clearance provided by the track channel is completely exhausted [mm].

For a tram wheelset designed for a gauge of $1000 \mathrm{~mm}$, with a wheel radius of $340 \mathrm{~mm}$, KP-1 profile, contact circles distance $2 S=1061.9 \mathrm{~mm}$ and value $\Delta r 5.5 \mathrm{~mm}$, the minimal value of the curve radius, that can be run theoretically without creep is $65.64 \mathrm{~m}$. For example, in Bratislava the city railways vehicles commonly operate on tracks with radiuses starting from $17 \mathrm{~m}$. It is therefore necessary to examine the options of increasing the $\Delta r$ value in formula (1). The relation of the desired $\Delta r$ value to the track radius can be expressed, based on the formula (1) as shown in Fig. 2. Increase in the $\Delta r$ value by increasing the value of the ride surface conicity cannot be seen as a suitable solution. Such intervention in the wheel profile geometry would lead to a more significant adulatory movement and thus worsening the vehicle ride stability at higher velocities. Thereby a conflicting requirement on increasing the $\Delta r$ value without intervening in the existing wheel profile ride surface geometry.

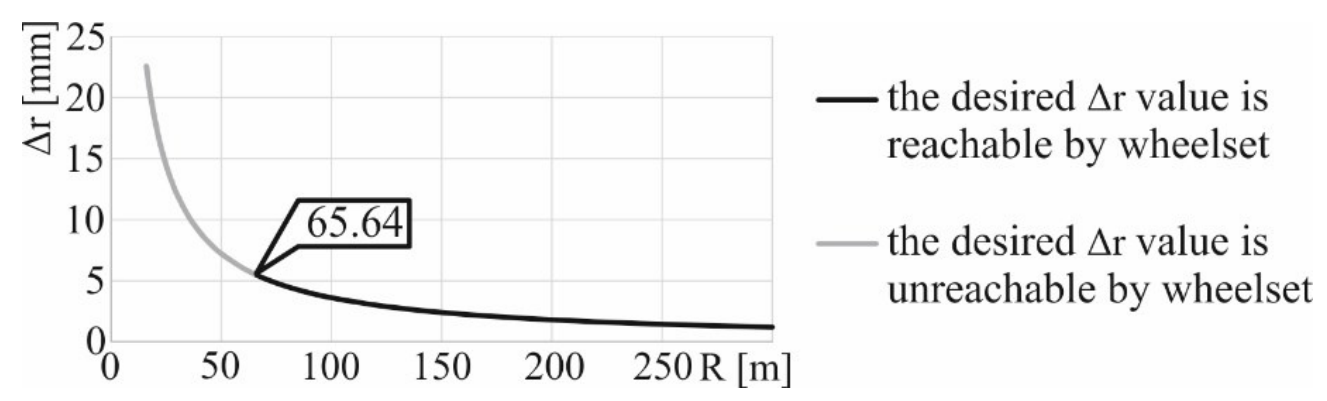

Fig. 2. Desired value $\Delta \mathrm{r}$ necessary for ride in curve without creep

Described conflicting requirement can be fulfilled by creating new point of rail-wheel contact, which will be used only for ride in strongly curved track.

\section{Suggestion of a way for wheelset passing a small radius curve}

We suggest solving this situation by using a wheel profile with an extended ride surface designed for a ride in small radius curves, located on the outer side of the wheel profile. A schematically picture of the wheelset with an extended profile and the principle of its ride through track curve is given in Fig. 3. 


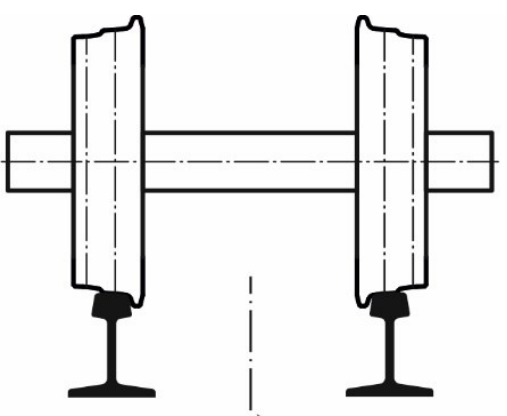

a)

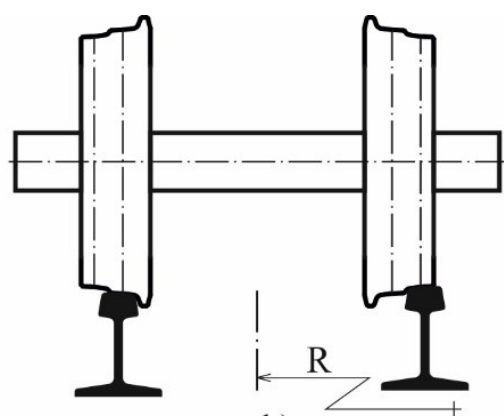

b)

Fig. 3. Wheelset equipped with an additional tread

a) during ride on a straight track, b) during ride in track arc of small radius

The inner part of the wheel profile is identical with the former profile. Ride characteristics of the vehicle during ride on a straight track or in curved track with a large radius therefore stays unchanged. An additional tread is designed on the outer part of the profile for ride of the axle on curved track with small radius. In curved track the inner wheel therefore rides on the additional tread of a smaller radius, allowing the required shift in delta $r$ function curve to be achieved.

For an effective use of the additional tread, it is necessary to determine its displacement from the original profile in lateral and vertical direction appropriately, defined using the dimensions $y$ and $z$ on Fig. 4a). For creating the geometry of the secondary tread, it is possible to use a segment of the original wheel tread.

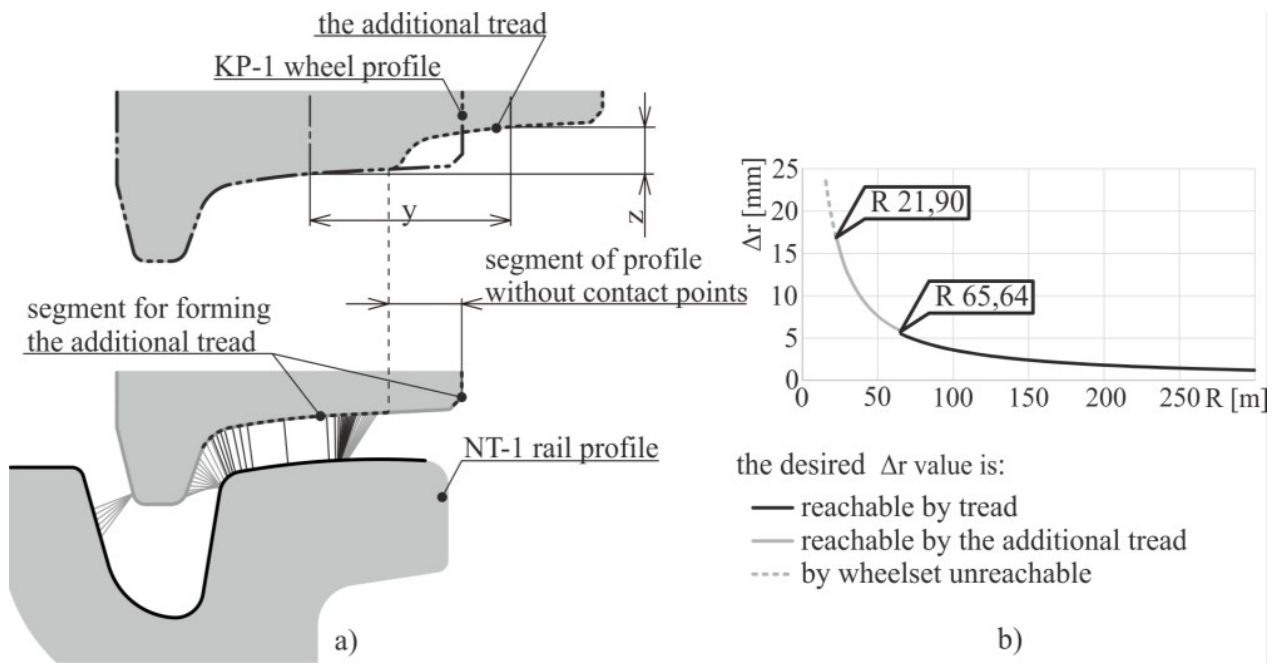

Fig. 4. a) Placement of the additional tread on the wheel profile,

b) Desired and attainable $\Delta r$ value for ride of the wheelset in curve without creep

The displacement in lateral direction should be minimal, to avoid significant increase in material volume and thus the mass of the wheel rim. However, its minimal value is limited by the distribution of the given wheel-rail pair contact points. For placing the additional tread, we suggest using the outer part of the original wheel profile, where the profile doesn't have any con-tact points with the rail. A certain widening of the wheel profile is necessary nevertheless. In the considered case of profile KP-1, a lateral displacement of the additional tread from the original one $y=50 \mathrm{~mm}$ was chosen. 
The displacement in vertical direction is dependent on the wheel profile delta $\mathrm{r}$ function value at the moment the clearance provided by the track channel is completely exhausted. For an effective use of the additional tread to be provided, following formula can be derived for the value $z$ :

$$
z=2 \Delta r+\frac{y \cdot \Delta r}{2 S}
$$

In the considered case of the original profile KP-1, the vertical displacement of the additional wheel tread value $z$ is $11.5 \mathrm{~mm}$. The required and achieved $\Delta \mathrm{r}$ value curve (as given in Fig. 2) is therefore changed in a way shown in Fig. 4b).

Based on the given theoretical consideration it is possible to expect more favourable ride in curve with a radius smaller than $65.64 \mathrm{~m}$ thanks to the use of an additional tread. A theoretical prerequisite for a wheelset ride without creep is created for a track curve radius of up to $21.9 \mathrm{~m}$. This theoretical assumption was verified by a simulation analysis of the vehicle's ride on a track with a described geometry. The contact geometry had to be inserted into the calculation program in the desired form.

\section{Creating inputs for simulation analysis}

To perform the simulation analysis, it is necessary to define, among other parameters, the wheel and rail profiles in the desired shape [12-14], i.e. in the form of text files containing among other parameters, particularly the sequence of coordinate points lying on the profile treads in the desired form. During the calculation, these points are automatically interconnected by the spline curve that represents the desired profile. For commonly used wheel and rail shapes, these files are already pre-written in the SIMPACK calculation program. The wheel-rail contact of the city railway vehicles is not covered by the standards concerning the railway vehicles, therefore the profiles needed for the following analysis cannot be found among the pre-written profile files. It was therefore necessary to proceed with their creation.

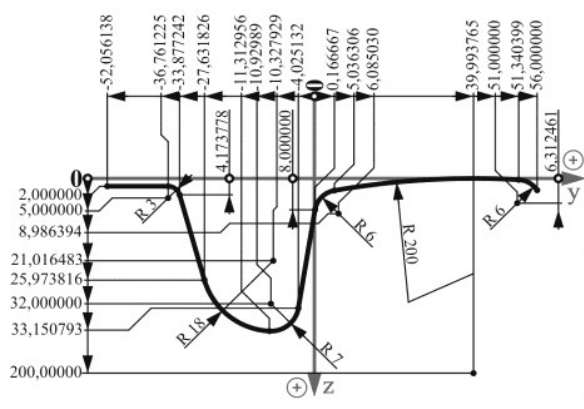

a)

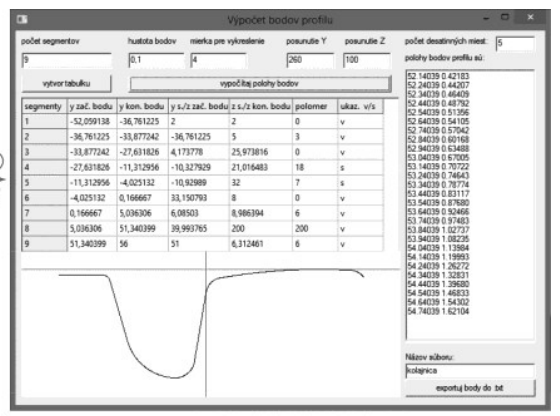

b)

Fig. 5. a) Drawing of the rail profile in desired shape,

b) Program dialog box for obtaining coordinate points

The required files can be added to the SIMPACK database, but SIMPACK does not have the ability to create such files directly by defining their geometry. It is therefore necessary to create the required sequence of points and define their coordinates in the appropriate file. The basic input for creating such file is the drawing of the profile in the desired shape, as shown in Fig. 5a). Based on this defined geometry, it is possible to create a set of parameter equations of the segments from which the profile is composed. 
Subsequently, based on the choice of one of the coordinate points, the second coordinate of this point can be counted using these parametric equations. This procedure must be repeated cyclically in order to obtain a sufficient number of points of the wheel profile tread. Approximately 200 to 3000 points evenly positioned over the wheel profile tread, depending on the profile complexity can be considered sufficient.

Because of the complicated process that is necessary to repeat many times when defining a rail with variable geometry, the authors have created a program containing algorithm, which allows to generate a sequence of coordinate points of the profile of arbitrary density based on a simple definition of certain specific dimensions of the individual profile segments, and save them in a form suitable for direct import into the SIMPACK program afterwards. A program dialog box with given specific dimensions of the rail tread segments generating the profile coordinates and plotting the specified profile is shown in Fig. 5b).

\section{Simulation analysis of a vehicle ride}

For verifying the theoretical prerequisite of wheelset ride without creep using the additional tread in track curves radius up 21.9 to $65.6 \mathrm{~m}$, as described in chapter 5, a series of vehicle ride dynamics simulation analysis was performed in simulation program SIMPACK with the aim of finding the dependency of the monitored quantities from the track curve radius. From among the examined quantities, the power dissipation arising from the vehicle ride resistance in track curve represents the effectiveness of the vehicle ride in curved track well. The curves of this quantity in three different situations are compared in this article.

In the first case, a vehicle without the possibility of steering the wheelsets nor the wheel profile with additional tread is considered. In the second case we consider a vehicle with a steering wheelsets possibility and without the proposed wheel profile design. The third case considers a vehicle with steering wheelsets equipped with the de-scribed new wheel profile design. The vehicle parameters basically resemble the ones of a T3 tram.

For obtaining an overall view of the vehicle behaviour it is necessary to evaluate the monitored quantities in dependence on the track curve radius. One of the ways to achieve such dependence is a simulation of vehicle ride along a track transition curve of a sufficient length. A track transition curve is a track section, where the radius of the curve is continuously changed. It can have various shapes. According to the TNŽ 736361 standard [15], a transition curve in the shape of a clothoid is allowed to be used in the track. In case the clothoid connects a straight track section with a circular curve, it is possible to calculate the values of its virtual radius $R[\mathrm{~m}]$ in the considered place with the use of the following formula:

$$
R=\frac{R_{2} \cdot l}{S}
$$

where $R$ is track curve virtual radius [m], $R_{2}$ is radius of the circular track curve on the end of the transition curve [m], $l$ is overall length of the transition curve $[\mathrm{m}]$ and $s$ is transition curve length measured from its beginning to the considered place $[\mathrm{m}]$.

In case the radius changes smoothly enough, the track section on which the vehicle is located currently can be considered as a curve with a constant radius. Therefore, the distance travelled along the track represented on the horizontal axis of the considered quantity curves can be calculated with the use of formula 3 for the track curve radius in the considered place. 

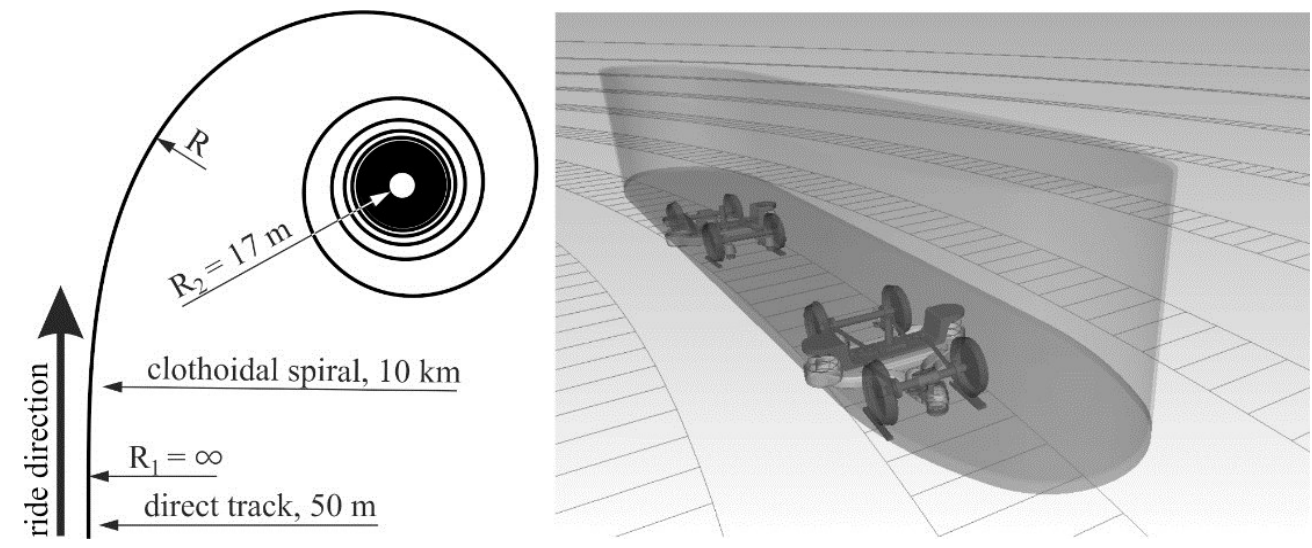

Fig. 6. Track in the shape of a transition curve

I the subsequent simulation, a ride on a track composed from a straight section designed for consolidation of the vehicle ride, a track transition curve in the shape of a clothoid with a length of $10 \mathrm{~km}$ and a circular arc with a radius of 17 metres. The shape of the track is shown in the Fig. 6. Comparison of the vehicle power dissipation during a ride at the speed of $10 \mathrm{~km} \cdot \mathrm{h}^{-1}$ is given in Fig. 7. The course of this quantity provides a global view for the way a vehicle rides in a track curve. Because of a big amount of the calculated values, only power dissipation curves for the curve radius interval from 17 to $100 \mathrm{~m}$, hence the area, where an effective application of the proposed design can be expected are presented. Power dissipation needed to cover the creep in longitudinal direction $\mathrm{P}_{\mathrm{x}}$ and in lateral direction $\mathrm{P}_{\mathrm{y}}$ are given separately, but the vehicle covers both power dissipations at the same time.
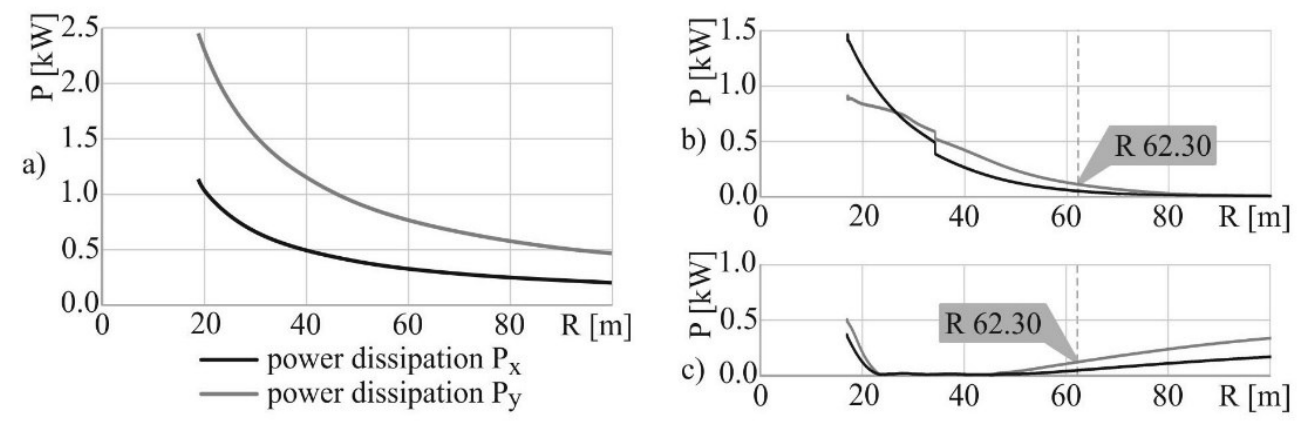

Fig. 7. Power dissipation of the vehicle

a) without the possibility to steer the wheelsets and additional tread,

b) with the possibility to steer the wheelsets and without additional tread,

c) with the possibility to steer the wheelsets and with the use of additional tread

Based on the simulation analysis of a ride in track transition curve it can be concluded, that in the case of vehicle ride in track curve with a small radius, it is possible to achieve more favorable ride parameters in a wider track curve radius interval.

From the comparison of the given simulation analysis can be evaluated, that a ride of a vehicle with use of an additional tread is more favourable in a curve with a radius smaller than $62.3 \mathrm{~m}$ in terms of power dissipation curves. From the given graphs it is evident, that it comes to significant reduce of negative effects in the monitored area when using the additional tread. In the track curve radius range of 23.55 to $44.88 \mathrm{~m}$ the power dissipation is practically eliminated. For the described way of vehicle ride in track curves with small 
radius to be able to be put to use, it is necessary to focus on creating an altered regime of wheel rolling during ride as well as examining vehicle response to such change.

It is generally known, that in terms of safety against derailment, the ride of the vehicle in an S-curved track with occurrence of superelevation twisting is seen to be very problematic. The goal of the authors was also to verify the vehicle's response to such situation that is further complicated by the change in the wheelset drive mode if the radius of the track exceeds the same value as the authors described in detail in Chapter 4. The track used for the calculation was defined according to TNŽ 736361 standard [15], consisting of straight segments, transition segments, and two curves of opposite direction with 25 meters radius. The length of track segments and their layout is shown in Fig. 8. The rails are seated with a lateral superelevation of $33.6 \mathrm{~mm}$. The track gauge is $1000 \mathrm{~mm}$.

We compare three cases: In first case the rails have a NT1 profile all along the track and the wheel profile was set to KP-1. Original bogie was used. In the other two cases the rail has a NT-1 profile on the straight section and a special shape (Fig. 8) in curve sections. In these cases, the wheel profile KP-1 with additional tread was used. Tram car was equipped with by authors proposed bogie [12]. Boogies layout facing each other with the front of the bogie frame and a facing each other with the frame rear was investigated. The part of the bogie frame with wheelset being seated in only one axlebox is considered the frame front.
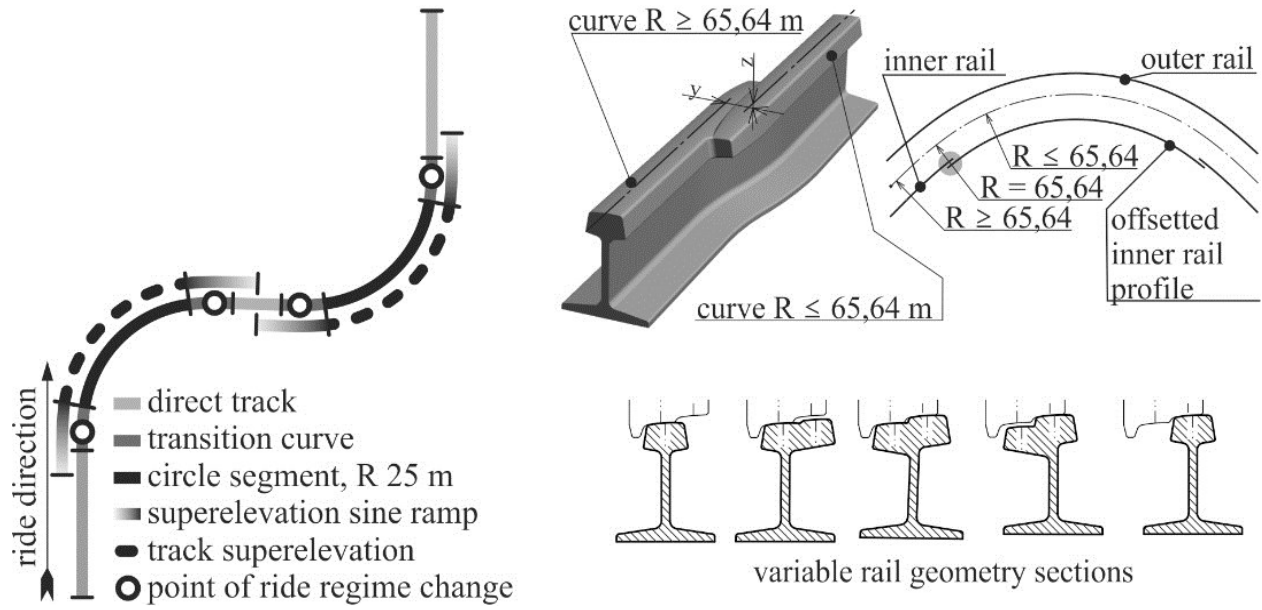

variable rail geometry sections

Fig. 8. S-curved track and a change point of driving mode

The tread change is performed on a wheel situated on the inner side of a track curve at the position of transition curve, where the curve radius measured from the track axis reaches a value of $65.64 \mathrm{~m}$. At this point, a change in the rail profile takes place. The rail profile placed in a curve of a small radius is offset against the original profile in lateral and vertical direction for a value identical with lateral and vertical displacement of the wheel profile. The change of wheel tread is performed through vehicle passing this part of the track, as is shown in Fig. 8.

When a wheelset enters a track curve, it attacks the outer rail. In a curve with a diameter corresponding the change of the tread, the clearance provided by the track free channel is considered to be completely reduced. If the change of the tread would be performed using an instant offset of the rail profile, as shown in Fig. 3, it would result in a step change of the $\Delta r$ value. The wheelset would suddenly get into a position favourable for a ride in curve with a radius of $21.9 \mathrm{~m}$, although the track radius in the given place is $65,64 \mathrm{~m}$, which would lead to violent attack on the rail. An instant offset of the rail profile would therefore become a source of lateral excitation, which is an unfavourable. It is therefore necessary to 
prevent a signifficant step change in $\Delta r$ value of the wheelset at the moment of tread change. This is reached using a varying rail profile near the place of tread change, which minimizes the step change of $\Delta r$ value.

The expression of the SAD (safety against derailment) as a ratio of guiding forces and wheel forces on individual wheels corresponds the guiding forces curve. Higher values represent a worse state, lower ones represent a safer ratio of horizontal and vertical forces. From the results in Fig. 9 can be seen, that all values are significantly under the generally known allowed value of 0.8. Higher values arise, when entering and coming out from the curve, but even in these cases they do not reach more than 0.5 .

a)
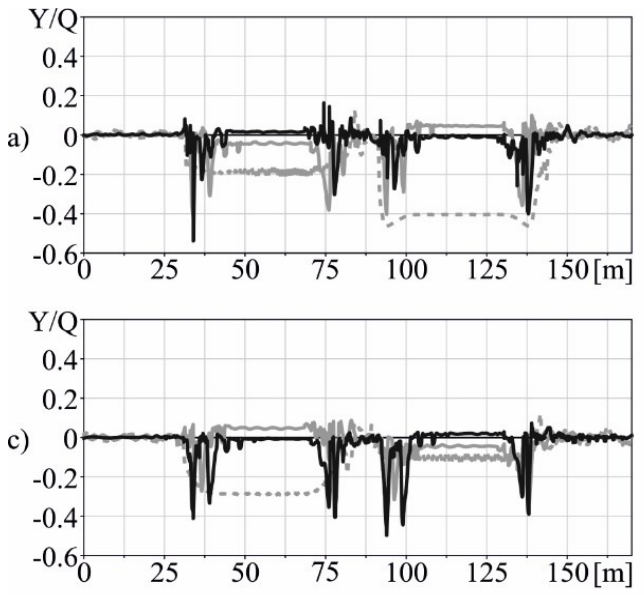

b)
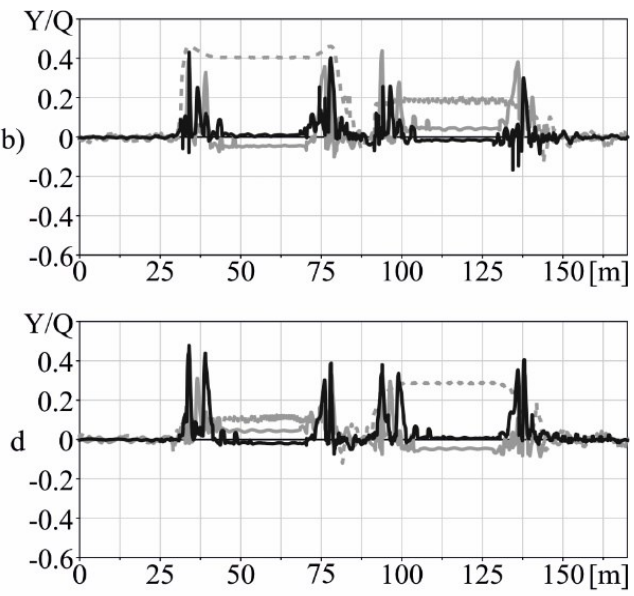

— with the bogies of the vehicle aligned with front parts facing each other

- with the bogies facing each other with the rear part

---- with original bogies

Fig. 9. Safety against derailment of the front bogie;

a) first wheelset, right wheel, b) first wheelset, left wheel,

c) second wheelset, right wheel, d) second wheelset, left wheel.

From the graphs can be concluded, that a tram with original bogies reaches worse results than bogies equipped with the steering mechanism. Bogies with the steering mechanism reach a maximal value of 0.05 and the original ones a value of 0.3 . At the moment of entering and leaving the curve, values around 0.5 are reached. The wheelset radial steering mechanism as well as usage of the additional tread have a positive impact on SAD.

\section{Conclusion}

Based on the simulation analysis of a ride in track transition curve it can be concluded, that in the case of vehicle ride in track curve with a small radius, it is possible to achieve more favourable ride parameters in a wider track curve radius interval.

From the comparison of the given simulation analysis can be evaluated, that a ride of a vehicle with use of an additional tread is more favourable in a curve with a radius smaller than $62.3 \mathrm{~m}$ in terms of power dissipation curves. From the given graphs it is evident, that it comes to significant reduce of negative effects in the monitored area when using the additional tread. In the track curve radius range of 23.55 to $44.88 \mathrm{~m}$ the power dissipation is practically eliminated.

For the described way of vehicle ride in track curves with small radius to be able to be put to use, an altered regime of wheel rolling during ride as well as examining vehicle 
response to such change was investigated. Rail of variable rail geometry was proposed. The wheelset running regime change possibility is able to ensure a more favourable ride in track curves. With its use, decrease of the SAD index, power dissipation and wear number during vehicle ride in track curves with small radius can be reached, as shown by the performed simulation analysis of a tram vehicle model with two different types of bogie.

The work was supported by the Cultural and Educational Grant Agency of the Ministry of Education of the Slovak Republic in project No. KEGA 077ŽU-4/2017: Modernization of the Vehicles and engines study program. The work was also supported by the project No. APVV-0842-11: Equivalent railway operation load simulator on the roller rig and VEGA Nr. 1/0927/15: Research of the use of alternative fuels and hybrid drives on traction vehicles with aim to reduce fuel consumption and air pollutants production.

Research-Educational Center of Rail Vehicles (VVCKV)

\section{References}

1. J. Dižo, Analysis of a goods wagon running on a railway test track. In: Manufacturing technology: journal for science, research and production 16 (4), 667-672

2. M. Blatnický, M. Štauderová, J. Dižo, Numerical analysis of the structure girder for vehicle axle scale calibration. Procedia Engineering 177, 510-515 (2017)

3. A. Chudzikiewicz, B. Sowinski, Modelling and simulation of trams bogies with fully independently rotating wheels. Dynamics of vehicles on roads and tracks, 1427-1434, ISBN 978-1-4987-7702-5, (2016)

4. A. Chudzikiewicz, Simulation of rail vehicle dynamics in MATLAB environment. Vehicle system dynamics 33 (2), 107-119, ISSN 0042-3114 (2000)

5. R. Melnik, S. Koziak, Rail vehicle suspension condition monitoring - approach and implementation. Journal vibroengineering, Lithuania, ISSN: 1392-8716

6. J. Gerlici, T. Lack, V. Hauser, M. Maňurová, O. S. Nozhenko, K. O. Kravchenko, Carriage bogie. Utility model no. UA 114040 U, category: B61F5/30, Kyjiv, Ukrajinskyj instytut promyslovoji vlasnosti (UKRPATENT), 9 p. (2017)

7. M. Loulová, V. Hauser, J. Gerlici, T. Lack, O. S. Nozhenko, O. P. Kravchenko, K. O. Kravchenko, Carriage bogie. Utility model application no. u201703246, category: B61F5/30, Kyjiv, Ukrajinskyj instytut promyslovoji vlasnosti (UKRPATENT), 9 p. (2017)

8. V. Hauser, J. Gerlici, T. Lack, M. Loulová, O. S. Nozhenko, K. O. Kravchenko, O. V. Prosvirova, Railway bogie wheelset and curved track segment. Patent application, number a201701589, category: 4267/3A/17, Kyjiv, Ukrajinskyj instytut promyslovoji vlasnosti (UKRPATENT), 11 p. (2017)

9. J. J. Kalker, Three-dimensional elastic bodies in rolling contact. ISBN 0-7923-0712-7, 314 p., 1990)

10. M. D. Ivanov, A. A. Ponomarev, B. K. M. Ieropol'skij, Tramways T-3. 240 p. (1977)

11. J. Zelenka, M. Kohout, The wheel - rail interaction in the environment of tramcar operation. [online] [cit. 2017-01-30] Available at: http://www.sdp-cr.cz/ WD_FileDownload.ashx?wd_systemtypeid=34\&wd_pk=W-zEzMDMsWzMwX (2012)

12. J. Dižo, J. Harušinec, M. Blatnický, Multibody system of a rail vehicle bogie with a flexible body. Manufacturing technology 15 (5), 781 - 788 
13. J. Dižo, M. Blatnický, B. Skočilasová, Computational modelling of the rail vehicle multibody system including flexible bodies. Communications: scientific letters of the University of Žilina 17 (3), 31-36 (2015)

14. L. Jakubovičová, M. Sága, Computational analysis of contact stress distribution in the case of mutual stewing of roller bearing rings. Novel Trends in Production Devices and Systems, Applied Mechanics and Materials 474, 363-368 (2014)

15. TNŽ 736361 Geometrical position and layout of railways with $1000 \mathrm{~mm}$ gauge. (ŽSR, Bratislava, 37 p., 2007) 